\title{
Prácticas alimenticias y clasificación social ¿Los tacos son un alimento "popular"?
}

\author{
Dietary practices and social classification
}

Are tacos a "popular" food?

Domingo García-Garza*

\begin{abstract}
Resumen: El consumo de tacos es revelador de la ambivalencia social que caracteriza las prácticas alimenticias contemporáneas. El análisis de un caso práctico arroja luz sobre el origen social y la modernidad de esta práctica alimenticia "popular". La perspectiva socio-histórica esclarece la manera en que el "taco" se aburguesó adquiriendo el rango de plato nacional. La interacción de fuentes de legitimidad favorece la divulgación de los tacos a todo el espacio social. La patrimonialización y la internacionalización del taco modificaron las representaciones de las prácticas populares. Ambos procesos transformaron la percepción y la naturaleza de las prácticas alimenticias en México. La observación etnográfica del consumo de tacos permite medir el peso de las fuerzas internas y externas que recaen sobre esta práctica para relativizar su clasificación social y repensar la producción de su legitimidad.
\end{abstract}

Palabras clave: Tacos; Alimentos; Prácticas; Consumo; Legitimidad

Abstract: The consumption of tacos reveals the social ambivalence that characterizes contemporary food practices. The analysis of a case study shows the social origins and the modernity of a "popular" dietary practice. The historical perspective highlights how tacos acquired the status of a national dietary practice through a gentrification process. The interaction of two sources of legitimacy helps to understand how these practices spread through the social space. The internationalization and "patrimonialization" of tacos modified "popular" food representations. Both processes thus transformed the perception and the nature of dietary practices in Mexico. The ethnographic observation of tacos consumption, which takes into account internal and external forces influencing this practice, helps us to rethink its social classification and to better understand the production of its legitimacy.

Keywords: Tacos; Food; Practices; Consumption; Legitimacy

* Maître de Conférences, Université Charles de Gaulle - Lille 3 (France). Investigador asociado, Centre Européen de Sociologie et de Science Politique (CESSP). École des hautes études en sciences sociales (EHESS).<domgarci@gmail.com>.

\begin{tabular}{|c|c|c|c|c|c|}
\hline Civitas & Porto Alegre & v. 10 & n. 3 & p. 430-449 & set.-dez. 2010 \\
\hline
\end{tabular}




\section{Introducción ${ }^{1}$}

Los tacos ${ }^{2}$ han sido considerados como un producto de origen popular. Efectivamente, su consumo representa una práctica alimenticia que nace en la parte más baja de la escala social. Su consumo se generaliza durante las primeras décadas del siglo XX, lo que coincide con la Revolución Mexicana (Pilcher, 2006a). Los tacos, al igual que otras prácticas culturales, se convirtieron en "emblemas nacionales" (Rivron, 2010) y han sido instrumentalizados para crear y reforzar la identidad nacional (Thiesse, 1999; Hubert, 2000; Bruegel \& Laurious, 2002; Pilcher, 1998). Sin embargo, el consumo del taco no se extendió a lo largo del espectro social sino hacia la mitad del siglo XX. La presente contribución trata de responder a la siguiente pregunta: ¿cómo un bocadillo de origen popular, plebeyo o campesino logró traspasar las fronteras sociales y erigirse como un platillo nacional?

Nuestra hipótesis es que el taco ha logrado extender su nicho de mercado tradicional porque experimentó un proceso de "aburguesamiento" a partir de la segunda mitad del siglo XX. Sugerimos que el taco se aburguesó por un doble proceso (patrimonialización e internacionalización) del cual se desprende una nueva legitimidad que le confirió otro estatus social. A partir del caso de tacos de "barbacoa" abordaremos la legitimidad obtenida gracias a la patrimonialización de la que fue objeto la comida popular. Y a partir del caso de los tacos "al pastor" mediremos la legitimidad de la comida mexicana inducida por la internacionalización. Gracias a estos dos casos prácticos, y a la forma en que se desarrolló el consumo de tacos, trataremos de aprehender el doble proceso de legitimidad que conoció la comida popular mexicana en general, y los tacos en particular.

Esta contribución es el resultado de una serie de investigaciones de campo efectuadas entre el año 2006 y el 2009 en la ciudad de Monterrey (México). Durante este periodo entrevisté a cerca de 60 taqueros y visité alrededor de 100 taquerías por las mañanas y por las noches. El método utilizado en esta encuesta fue la etnografía (Beaud \& Weber, 2003; Weber, 2009), es decir, una combinación de métodos como la entrevista formal e informal, la observación participante, así como la consulta de archivos históricos y periodísticos para estudiar un caso práctico. La ambición de este artículo es medir la forma en que las representaciones y las percepciones de un alimento

Agradezco a Mauricio Bustamante y a Fruela Fernández, del Centro Europeo de Sociología (CSE-EHESS), por sus valiosas observaciones y comentarios.

2 Tortilla de maíz enrollada con algún alimento dentro, típica de México (Real Academia de la Lengua Española). 
modificaron su consumo. Evocamos tangencialmente el doble pasaje de la esfera doméstica a la esfera mercantil (Weber \& Dufy, 2008); y el pasaje de la venta a granel a la venta dosificada en forma de tacos (García, en prensa). Ambos fenómenos explican la aparición del mercado de tacos contemporáneo. Sin embargo, aquí nos concentraremos especialmente en la manera en que se operó la revalorización social de esta práctica alimenticia a través de la patrimonialización y posteriormente, su internacionalización.

\section{La legitimación de la comida "popular" mexicana}

Partimos de la idea que la llamada comida "popular" mexicana se legitima por dos procesos que pudieran parecer antagónicos (patrimonialización/ internacionalización), pero que terminan siendo complementarios. El primero es interno y evidencia la forma en que los diferentes terruños del país contribuyeron a definir la "cocina nacional mexicana" gracias, entre a otras cosas, a nuevos espacios sociales como las taquerías (Pilcher, 2006a, p. 90). El segundo proceso es externo e ilustra la manera como los bienes culturales se legitiman por la forma en que circulan por los mercados transnacionales (Heilbron, 2001) ${ }^{3}$. La transnacionalización de la comida mexicana tuvo lugar gracias a la migración de mexicanos a Estados Unidos y la exportación consecuente de la comida mexicana al vecino país del norte. El caso de los tacos es revelador del proceso de revalorización de la imagen de un producto popular de origen nacional gracias a su pasaje por el extranjero. De ahí que se explique en parte la expansión del consumo de tacos a todo el espectro social en México.

Los tacos eran calificados como un producto "popular" y su consumo sufría del estigma de ser una práctica alimenticia propia a personas de escasos recursos. Estas prácticas alimenticias, y el producto mismo, han sufrido una serie de transformaciones que condujeron con el tiempo a su aburguesamiento. El aburguesamiento del taco no puede explicarse únicamente por el hecho que los tacos hayan llegado a la mesa de los consumidores socialmente más favorecidos. La generalización de su consumo se podría explicar por la apreciación que experimentó este bocadillo a la luz del proceso de construcción nacional y de internacionalización de la comida mexicana. El taco experimenta por un lado un proceso de patrimonialización (Heinich, 2009) culinaria que

\footnotetext{
3 Para apoyar nuestra hipótesis sobre el aburguesamiento del taco nos basamos en el marco teórico propuesto por Heilbron (2001). En él se establece que la circulación internacional de bienes culturales depende de la posición de los países por donde circulan dichos bienes y de la centralidad (o periferia), tanto cultural como política y económica, que ocupan los países en el sistema mundial de intercambios.
} 
modifica su estatus en la jerarquía gastronómica nacional adquiriendo el rango de patrimonio vivo de la nación. La difusión del consumo de tacos en un país central como Estados Unidos por el otro, permitió a los tacos adquirir una mayor visibilidad en ambos países. Los dos procesos anteriores cambiaron la naturaleza social del taco en México confiriéndole un mayor prestigio. Es justamente este doble proceso el que contribuyó a la difusión de productos y de prácticas "populares" en todo el espacio social mexicano.

\section{La patrimonialización de la comida mexicana: los tacos de barbacoa}

A partir del caso de los tacos de barbacoa (carne de res cocida al vapor) trataremos de aprehender el aburguesamiento de esta práctica alimenticia a través de la "patrimonialización" de la comida regional. Este ejemplo nos permite observar cuál fue la evolución que conoció el mercado de los tacos en general durante la segunda mitad del siglo XX. El consumo de tacos de barbacoa ilustra la manera en que una práctica popular asociada a la pobreza pasó a ser una práctica alimenticia generalizada entre la población.

La carne cocida al estilo "barbacoa" se obtiene gracias a una antigua técnica de cocimiento milenaria (Fournier, 1999, p. 459) muy difundida en la llamada Huasteca Potosina (centro del país). Dicha práctica se divulgó en Monterrey desde principios del siglo XX.

Sr. Lencho alias el "Cumbia King" (vendedor de tacos de barbacoa de cabeza): ¡Soy "regio"”, de corazón! Nací en la Colonia Independencia (una de las más populares de la ciudad, llamada también "San Luisito" por estar poblada esencialmente por gente del vecino estado de San Luís Potosí). Nosotros venimos de Don Silvestre Morales (padre de Don Julián).

Sr. Julián Morales (dueño del "cocedor” de barbacoa): Mi padre es [era] de la Huasteca, del Rancho "El Panalillo". Papá trajo mucha gente de allá. Había mucha gente San Luís Potosí. [La barbacoa] así es como hacían la carne allá.

Todos los testimonios coinciden en que, al menos en México, la tradición de cocer la carne al estilo barbacoa proviene del estado de San Luís Potosí. Dicha técnica fue traída por los emigrantes del vecino estado que se establecieron en Nuevo León. La tradición de la barbacoa se adoptó paulatinamente en toda la región del noreste y particularmente en Monterrey. Entre los primeros consumidores de barbacoa figuran naturalmente los miembros de la misma

\footnotetext{
4 "Regio" es la contracción de "regiomontano", el gentilicio o nombre que reciben los habitantes de Monterrey.
} 
comunidad provenientes de San Luís Potosí. El consumo de tacos ilustra los lazos que los habitantes de la región, originarios de San Luís, mantienen con su terruño de origen o su "patria chica" (Pilcher, 2006a). Los autóctonos de Monterrey fueron adoptando paulatinamente la técnica como especialidad local y en esa medida se "inventó la tradición" de la barbacoa (Hobsbawn \& Ranger, 1992). Merece la pena subrayar que incluso los restaurantes más exclusivos de la ciudad sirven por las mañanas la tradicional barbacoa para el desayuno.

No hay que confundir sin embargo la barbacoa con la parrillada, el asado, el grill o la barbecue anglosajona, (que son variantes pragmáticas del método tradicional de origen precolombino). Por requerir una preparación determinada y cierto tiempo, la barbacoa se destinaba a ocasiones especiales (bodas, quince años, bautizos, etc.). En efecto, la barbacoa era catalogada como un platillo ceremonial.

Sr. Julián Morales: Todo empezó en los 1930s. Por lo que yo sé. Porque los pozos de antes, era con leña que les prendíamos lumbre. [Llenábamos los pozos] con leña, metíamos pencas [hojas de agave o maguey], costales y después los cerrábamos con una capa de tierra [los pozos fueron prohibidos en los años 60s por las autoridades de Salud Pública]. La barbacoa era para bodas, bautizos, todo eso. Pero aquí, jera cosa de todos los días! Los muchachos iban a vender [barbacoa] a la calle, todos los días, en un canasto.

Observamos que con el tiempo el consumo de la barbacoa se banalizó. La barbacoa se convirtió, después de su uso ritual como comida de pasaje a diferentes edades sociales, en uno de los platos dominicales por excelencia en el centro y en el norte de México. Poco a poco se empezó a vender este tipo de carne los domingos en las carnicerías locales. Su consumo era doméstico y tendía a preservar la unidad familiar. Según los testimonios y los archivos municipales la barbacoa se comercializaba en las calles desde mucho tiempo atrás. Los llamados "barbacoyeros" (quiénes cocían la carne a la barbacoa) de la Colonia Independencia reproducían la tradición de su tierra natal para cocer carne y la vendían desde finales del siglo XIX alrededor de la antigua estación del tren (Mercado del Norte), en las colonias populares, alrededor de la Basílica de Guadalupe y desde principios del siglo 20, en el centro de la ciudad5. La barbacoa era distribuida por los llamados "corredores" que la repartían a pie por la ciudad.

\footnotetext{
Archivo Histórico de Monterrey (AHM), sección de Comercio y Pisos, años 1881-1889.
} 
Sr. Armando Gutiérrez (vendedor de barbacoa): Había mucha gente de San Luís [Potosí]. Don Isidro Narváez, trajo muchos "corredores" de su pueblo, para vender barbacoa en las calles. Eran corredores, con canasto. Metían la carne en un canasto y se iban pa'abajo, pa'alla, pa'la ciudad, a vender. [Con] una o dos cabecitas [de res].

Los "corredores" (distribuidores al menudeo de carne) bajaban la colina donde está enclavada la Colonia Independencia (donde los "barbacoyeros" cocían la carne) y atravesaban el Río Santa Catarina (principal frontera geográfica y social) para llegar al centro. El centro es el "mercado natural" de tacos por congregar diferentes instituciones, tanto públicas como privadas; además de ser un modo de comunicación por donde pasan prácticamente todas las rutas de autobuses urbanos. El centro de la ciudad es también el lugar de convivencia y mezcla social más importante de la ciudad. Fueron las personas originarias de San Luís instaladas en Monterrey las que participaron a la difusión de la cultura del taco de barbacoa en la ciudad.

Sr. Armando Gutiérrez: Yo vendo tacos [de barbacoa] desde 1967, aquí, en la Independencia. Esto es herencia de mi padre. Nosotros seguimos su camino. Yo nací aquí, pero mi padre (Melesio Gutiérrez) era de San Luís [Potosí]. El, Silvestre Morales, Isidro Narváez, Agapito Gutiérrez... Bárbaro, Pascual, todos esos... Todos lo que vendían en el Mercado del Norte, en el Mercado Colón... Todos ellos son de San Luís [Potosí]. Mi padre llegó a la edad de 15 años. Se casó a los 17 [años]. Era barbacoyero.

La venta ambulante de barbacoa en las calles de la ciudad es una tradición que data desde finales del siglo XIX y está bien documentada en los Archivos Municipales de Monterrey (AHM). La barbacoa era preparada en sus inicios a partir de la llamada "carne chica", es decir carne de origen caprino y ovino. En la década de los años 1950s la comunidad de "barbacoyeros", instalados en la (popular) Colonia Independencia, comenzó a introducir progresivamente carne de origen bovino.

Sr. Armando Gutiérrez: Se vendía barbacoa. De canasto. Era, barbacoa, como ahora. Pero antes era de "carne chica", de borrego o de cabrito. Porqué sólo había eso. La gente sólo comía eso. Carne típica de la región. Después empezaron a meter [carne] de res, cabeza de res.

La carne de res representaba una alternativa más rentable con respecto a la carne de cabrito y de borrego (que era más cara). El precio de la carne de res es más competitivo que las otras ofertas cárnicas y presenta un mayor rendimiento. Quizás por el hecho de que su sabor sea menos fuerte que el de 
la carne de borrego, otros grupos sociales fueron aceptando paulatinamente la barbacoa de res.

Sr. Armando Gutiérrez: En 1952-53, empezamos a vender otros tipos de carne. Yo mismo comencé a vender en ese año [carne de res]. Vendíamos labio, jeta, cabeza de res. Todo eso. Desde entonces.

En la ciudad se comercializa hoy en día prácticamente barbacoa de res. Contrariamente a otras regiones del país, en el norte de México existe una tendencia a cocer únicamente la cabeza de la res. Los tacos de barbacoa que se comercializan en Monterrey son casi exclusivamente de cabeza de res. Todas las partes de la cabeza son apreciadas por los consumidores de tacos, especialmente los sesos, los ojos, el paladar, la lengua y evidentemente el cachete o labio. Sin embargo la barbacoa no se vendía en forma de tacos antes de los años 1950.

Sr. Armando Gutiérrez: En ese entonces se vendía carne, así nomás, en plato [a granel]. No eran tacos. ¡No! Lo de los tacos tiene poco. Hará hace 30-40 años, no sé.

El testimonio del Sr. Gutiérrez nos deja ver la evolución de la venta de barbacoa al menudeo. La venta de barbacoa se vendía anteriormente a granel y desde mediados del siglo XX se vende por taco (o por unidad). La introducción de la tortilla industrial en la década de los 1950s fue determinante para que se consolidara el mercado de tacos contemporáneo.

El consumo de tacos de barbacoa, que es el producto de una técnica ancestral, ilustra la forma en que las diferentes apropiaciones sociales de una práctica cultural favorecen la formación de un imaginario común (Anderson, 1991). Profundamente anclados en las prácticas alimenticias nacionales y en el imaginario popular, los tacos participan a la definición de la identidad social, al mismo tiempo que el taco se integra de forma no concertada al panteón nacional contribuyendo a la patrimonialización culinaria, generada por el consumo del taco en todos grupos sociales y por su arraigamiento en la historia nacional de México.

\section{Los tacos y la morfología social de Monterrey}

¿Cómo se difundió el consumo de tacos "populares" entre la población? Todo indica que las taquerías callejeras jugaron un rol determinante en la popularización de los tacos de barbacoa en Monterrey (García, 2009). Las taquerías creadas en la década de los 1980-90s contribuyeron en gran medida a la difusión de la "cultura del taco" más allá de su nicho de mercado natural (clase baja, obreros, empleados, albañiles). Lo anterior no significa que 
no hayan existido taquerías en Monterrey. Prácticamente sólo existían tres taquerías instaladas en locales comerciales (La Taquería Juárez, La Mexicana y La Rosa Náutica). No es sino hasta la década de los años 1980s que explota el número de puestos de tacos en la calle. Las taquerías callejeras se adaptan perfectamente a la nueva configuración económica de la llamada "década perdida" (crisis, contracción del mercado de trabajo, inflación, estancamiento de salarios, etc.). No es un azar que con la reducción del poder adquisitivo, los consumidores optaran por alternativas alimenticias más económicas como los tacos callejeros.

Entre las primeras taquerías que aprovecharon deliberada o indeliberadamente la misma clientela de las discotecas figuran Gyros n' Tacos, Tacos del Julio, El Rincón Sonorense y El Pirata Memo en San Pedro (como lo veremos más tarde); y Tacos Don Nacho en Monterrey. El Sr. Ignacio Medrano, alias "Don Nacho", es uno de los taqueros de mayor éxito en la ciudad. Es originario de Hidalgo (Tamaulipas) y vende tacos desde 1987 enfrente de la Alameda Central (esquina de las calles Rayón y Arramberri).

Sr. Ignacio Medrano (vendedor de tacos de barbacoa de labio de novillo): Pues yo era chofer de ruta [autobús urbano]. Y un señor que vendía tacos [de barbacoa], al que yo le "feriaba" [cambiar los billetes por monedas], me decía que me "desencadenara", que me bajara del camión [autobús], que los tacos dejaban más billetes [eran mas rentables]. Él fue el que me enseñó a trabajar. ¡[Me dijo] que metiera labio de novillo de primera calidad! Y que, pasara lo que pasara, nunca metiera otra cosa [otro tipo de carne]. Ve a ver lo que venden ahí en el Obelisco pa'que veas... ahí en los puesteros...

El caso del Sr. Medrano es representativo de las mutaciones del mercado alimenticio. Ya que sin saberlo opera un cambio significativo al sustituir la carne de cabeza por "labio" o cachete de res. Por insignificante que parezca, su éxito radica en parte en este aspecto. La carne de labio de novillo de res no sólo es de mejor calidad organoléptica o gustativa, sino que es menos grasosa que los otros bocados que componen la cabeza de res. Los tacos hechos con carne de labio de res tienen más posibilidades de llegar a un público más amplio (joven, acomodado, cosmopolita) porque se adecuan mejor con las representaciones sociales de la alimentación contemporánea (menos ricas en grasa de origen animal). El reemplazo de la cabeza de res (carne mexicana fresca) por el labio de novillo de res (carne estadounidense congelada) le permite conquistar otros nichos de mercado que antes no consumían barbacoa ${ }^{6}$.

6 La oposición entre la carne fresca (mexicana) y la carne congelada (norteamericana) ilustra las luchas históricas entre los principales actores de la distribución de carne en México (Pilcher, 2006b). 
Aunado a la popularización de la barbacoa de labio de novillo, el mercado de los tacos aprovecha la aparición de los llamados antros ${ }^{7}$ (bares, discotecas). Las taquerías que aparecen en los 1990s comienzan a captar a los clientes que frecuentan los recién creados lugares de esparcimiento para los jóvenes. Estos constituyen un nuevo espacio de socialización que incrementa la demanda de alimentos. Entre los primeros antros de la ciudad figuran el Sargento Pimienta y el Noctis en San Pedro, así como el Café Iguana, el Escena y el Kokoloco en Monterrey. Lo que no quiere decir que las taquerías hayan nacido gracias a la creación de los antros. En efecto, las taquerías precedieron a los antros. Sin embargo, la aparición de estos últimos estimuló indirectamente el mercado de tacos en ese periodo.

Las taquerías callejeras tienden a paliar la limitada oferta alimenticia nocturna y a reducir el costo de alimentación comparado con el de otras alternativas disponibles (restaurantes para trasnochadores). Las taquerías de la calle son atractivas porque sus costos son menores, porque no exigen las mismas reglas de etiqueta que los restaurantes y porque acortan significativamente los desplazamientos al situarse cerca de las discotecas. Los puestos de tacos "conocidos" minimizan el riesgo que implicaría consumir alimentos cuya calidad se desconoce y reducen al mismo tiempo el costo que implicaría desplazarse a otros puestos localizados más lejos. La clientela tradicional de las taquerías callejeras fue cambiando conforme los empleados y luego los clientes de los antros y discotecas empezaron a frecuentar los puestos callejeros de tacos regularmente. Los nuevos consumidores generaron, por efecto "bola de nieve", la atracción de fracciones de población con un perfil socio-económico más elevado. Aunado a la clientela habitual, los nuevos clientes aumentaron significativamente el volumen de ventas de las taquerías. Este pequeño grupo social actuó como caja de resonancia y contribuyó a expandir el consumo del taco en otros círculos renovando su imagen entre los grupos sociales que no lo consumía forzosamente. El siguiente extracto de entrevista deja ver la manera en que fue cambiando la clientela de las taquerías callejeras.

Domingo: ¿Cómo fue llegando la "raza” [jóvenes] a su puesto Don Nacho?

Sr. Nacho: Los [empleados] de las "discos". Los que jalaban [trabajaban] ahí. Cuando salían [de trabajar por la madrugada] se venían pa'aca, a "echarse" [comerse] un taco. Y luego fueron llegando también los clientes [de las discotecas]. Y así. Toda la gente de los bares, de los lugares esos... ¿cómo se llaman...? ¡Los antros! 7 Local, establecimiento o vivienda de mal aspecto o reputación (Real Academia de Lengua
Española). 
Domingo: ¿Cuáles antros?

Sr. Nacho: Pos esos de ahí... Que el Escena, que el Reloj... los de ahí, los del "Barrio Antiguo" y otros más, no sé ni cuales...

La aparición de estos establecimientos de diversión en los años 1990s coincide con un relajamiento de los hábitos de consumo en general y de las prácticas alimenticias en particular. Todo indica que existe una correlación entre la economía del "relajo" o del ocio que representan los antros y la economía alimenticia (García, 2005). El nuevo apelativo de las discotecas, "antros", es revelador del debilitamiento de las convenciones sociales. El auge del consumo de tacos parece ser la expresión culinaria de una distensión social más amplia. Por situarse a las antípodas de la configuración del restaurante clásico, la ingesta de tacos en la calle se acomoda perfectamente con las nuevas tendencias del consumo. Una clientela variopinta encuentra en las taquerías callejeras una alternativa que reemplaza el conservadurismo y el apego a los ritos del consumo alimenticio tradicional (ingesta en casa, horarios fijos, con cubiertos, sentados). Las ingestas callejeras ilustran en ese sentido la llamada destructuración alimenticia. Los tacos callejeros, como cualquier otra comida ingerida fuera de casa, corresponden a la demanda de individuos "desarraigados", sometidos a los imperativos de la vida moderna (Fournier, 1999, p. 470).

Los regiomontanos no son los únicos que contribuyeron a la disolución de las convenciones del consumo alimenticio vigentes. La comunidad de estudiantes llamados "foráneos" (de otras partes del país), contribuyó fuertemente al desarrollo de una nueva cultura urbana más "relajada". La población estudiantil que reside en la ciudad durante gran parte del año constituye una parte significativa de la clientela de los antros y de las taquerías. Los tacos son una alternativa seductora para satisfacer las necesidades de alimentación de la comunidad de estudiantes universitarios locales y foráneos.

La presencia de estudiantes foráneos en Monterrey desencadenó una serie de actividades económicas ligadas a su estancia en la ciudad. Con cuatro grandes universidades, una pública (UANL) y tres privadas (ITESM, UDEM, UR ${ }^{8}$, Monterrey es un polo educativo importante de la República Mexicana. Entre dichas actividades figuran principalmente el alojamiento, pero también las actividades de ocio (discos y antros) y de alimentación; sin olvidar el mismo mercado educativo que ellas representan. El mercado alimenticio que se

8 Universidad Autónoma de Nuevo León, Instituto Tecnológico y de Estudios Superiores de Monterrey, también llamado "Tec"; Universidad de Monterrey y Universidad Regiomontana, respectivamente. 
desarrolló alrededor del ITESM al sur de la ciudad es una de las manifestaciones más visibles (el ejemplo más emblemático es la ahora desaparecida "Calle del Taco" situada en la Privada Filósofos en la Colonia Tecnológico). En dicha área floreció una nutrida oferta alimenticia desde la década de los 1980s como por ejemplo: Tacos Félix, Tacos El Güero, El Rincón Sonorense, El Arbolito, Tacos El Carnal, etc. Los archivos de la ciudad dan cuenta del mismo fenómeno con respecto a la década de los 1980s cuando explotó el universo de taquerías informales que contribuyeron a banalizar su consumo entre los jóvenes de la ciudad. El fenómeno se convirtió en un "problema social" que se solucionó en los 1990s bajo la administración municipal del conservador Partido de Acción Nacional (PAN). Sin embargo, la moda de los tacos se instauró durante ese periodo expandiéndose a otras zonas de la ciudad y a otros grupos sociales.

Otro de los factores que ayudaron a que el taco se expanda a otros grupos sociales fue la aparición de taquerías en los barrios residenciales que se crearon en el noroeste de la ciudad en los años 1960s (entre los cuales figuran las colonias Mitras, Vista Hermosa y Cumbres).

Domingo: ¿En qué año empezó a vender tacos [al pastor] en Monterrey?

Sr. Julio Reyna (Taquería La Playita): Vamos a ponerle, ¡1964! Nosotros llegamos en 1962, digamos que empezamos dos años después de haber llegado aquí [a la Colonia Mitras Centro].

Domingo: ¿Creo que fue su padre el que empezó a vender tacos aquí?

Sr. Gustavo Lara (Tacos Lara): ¡[El negocio] lo empezó mi papá! Pero de hecho, mi papá no conocía los tacos. Mi papá, cuando llegó de Zacatecas, no tenía trabajo. Pasó por un negocio, una tortillería que decía: "Contrato persona que venda tacos. Doy el $25 \%$ de comisión”. Entonces, mi papá empezó a trabajar ahí. Al poco tiempo, venía él en la bicicleta por ahí, por [avenida] Madero, y se puso por aquí, en [la avenida] Gonzalitos y [Calzada] Madero. Antes había una tienda [supermercado] que se llamaba Azcúnaga. Y se paró ahí, por el cansancio, o porque ahí se quería parar. Y empezó a acercarse la gente. ¡Ahí hizo punto! Y aquí en el local tenemos establecidos nueve años, desde 1998. Del 1976 al 88, estuvimos en una moto. Del 1988 al 96, estuvimos en una camioneta. Y del 1997 estamos establecidos en este local [en la Colonia Vista Hermosa].

Las dos entrevistas anteriores atestiguan que las taquerías aparecieron en la década de los 1960s en los recién fraccionados sectores al poniente de la ciudad. Ambas colonias, Mitras y Vista Hermosa, surgen en ese periodo al acelerarse la urbanización hacia el norte y el noroeste de la ciudad. Estas 
colonias son consideradas como áreas "residenciales" y se fueron poblando por la creciente "clase media". Las nuevas fracciones en ascenso social provenían generalmente del interior del Estado de Nuevo León, cuya capital es Monterrey, de otras partes de la República Mexicana, o bien, de barrios abandonados por sus antiguos habitantes a expensas de los recién llegados (el centro de la ciudad). Los nuevos habitantes de estas áreas fueron adoptando el consumo de tacos en taquerías de tipo restaurante.

El mismo fenómeno se repite en el llamado "Centrito Valle" que es la zona comercial de San Pedro Garza García (exclusivo suburbio al poniente de Monterrey). En la ciudad de San Pedro, y de forma paralela a la aparición de los antros, aparecen varios expendios de tacos en la década de los 1980s.

Domingo: ¿Cuál fue la génesis de los tacos aquí en San Pedro [Garza García]?

Sr. César Rodríguez alias “El Gordo" (Gyros n' Tacos): Pues todo empezó a raíz de las “discos” [discotecas]. Alguien vio la necesidad de los que [los jóvenes] salían de la disco y querían echar[se] un taco. Y hace 17 años, [el dueño de Gyros n' Tacos] vio esta idea en Estados Unidos, en Chicago. Y la quiso adaptar para la gente que iba a saliendo del "antro". Y pues empezó a funcionar lento, lento. Al principio eran él y su esposa. Y conforme las necesidades de... espacio y todo. Se fue buscando un espacio más amplio, más mesas, más personal... Hasta que mi patrón se deslindó de esto y pues yo quedé a cargo del negocio.

Domingo: ¿Cuáles fueron las primeras "discos”?

Sr. César Rodríguez: No, pues no recuerdo. Pero se menciona que fueron "El Sargento Pimienta”... ¿Cuál otra era? Una, ahí donde está el "Alebrije" ahora.

Domingo: ¿Hace 17 años de esto? ¿Cómo empezó? ¿Con un puestito?

Sr. César Rodríguez: Mi patrón empezó con un carrito pequeño. Luego puso un puentecito ahí, donde era la "Cabaña de Don Quintín”. Ahora es el Ocean Drive, ahí. Y pues una vez se quemó todo. Y pues sacaron a mi patrón de ahí también. Y mi patrón, vino y habló con el Sr. Modesto Hernández. Y le rentó este espacio [sobre la calle Río Orinoco de la Colonia del Valle].

Domingo: ¿Qué discos estaban ahí?

Sr. César Rodríguez: El "Noctis", ahora es el "Ilumen".

Domingo: ¿Y antes de eso, qué? ¿No había discos, no había nada?

Sr. César Rodríguez: No, no creo. No recuerdo.

Todos los testimonios y archivos coinciden en un punto: si bien las taquerías callejeras ya existían, muchas otras nacieron con la aparición de 
los antros y discotecas. Ambos negocios buscan la misma clientela (jóvenes, estudiantes universitarios). Sin embargo, hasta los años 1980s sólo había un par de discotecas y presentaban uno o varios estigmas ligados a la clientela que a ellas asistía (de clase social, de localización, en fin, étnicos). La "clase media" que ayudó a difundir el consumo de tacos en todo el espectro social frecuentaba generalmente los bailes organizados en el Club de Leones (Colonia Mitras), alrededor de la Iglesia de La Purísima (zona poniente del centro) y la calle Morelos (centro). Monterrey era efectivamente una pequeña ciudad en expansión y las opciones de alimentación y diversión eran reducidas. La aparición de los antros adaptados a la clase media emergente contribuye a banalizar el consumo de tacos. El taquear (comer tacos) se impone en el lenguaje de los clientes jóvenes y en las prácticas de consumo gracias a la mediación de grupos sociales más propensos a franquear las convenciones que rigen la conducta y los hábitos alimenticios.

Observamos que desde sus inicios, algunas taquerías nacen tratando de aprovechar el flujo de personas, generado por otras instituciones, como por ejemplo los "Tacos Rojos" de la familia Nuncio, que se instalaron enfrente de las Oficinas del Registro Civil a principios del siglo XX. Sucede lo mismo con las taquerías aledañas a las discotecas, que buscan captar una parte de la clientela de éstas. Nótese que las taquerías dependen de las discotecas y que ambas viven en una especie de simbiosis económica gracias en cierta medida a la comunidad de estudiantes, a los noctámbulos, los trasnochadores, etc. Sin embargo, el éxito de las taquerías depende del éxito de los antros y de la nueva morfología social y urbana de la ciudad de Monterrey.

Sr. César Rodríguez: Si. Aquí el mercado va enfocado a los jóvenes que vienen a los antros. Y la mayoría de la clientela que tengo yo, ¡son foráneos [de otras partes del país]! ¡Influye mucho! En los periodos de vacaciones en el Tecnológico (ITESM), en la UDEM, [el volumen de ventas] bajo mucho.

Una regularidad es que la nueva ola de taquerías nació alrededor de los recién creados antros y que los estudiantes "foráneos" inscritos en las universidades privadas constituyen un nicho de mercado importante para las taquerías. La entrevista deja entrever que el mercado alimenticio evocado se desarrolla en la zona del Centrito Valle, localizada en el suburbio de San Pedro. Muchos estudiantes foráneos viven en este sector, que además de ser una de las más exclusivas de la Zona Metropolitana de Monterrey, es el municipio con el mayor PIB per capita del país y donde está ubicada la Universidad de Monterrey (UDEM). El nivel de vida de San Pedro es acorde con el estatus social de origen de los estudiantes de las universidades privadas 
y el relajamiento de las prácticas de consumo se da con mayor facilidad en un contexto como éste. El consumo de tacos en una configuración diferente elimina los estigmas que caracterizaban a esta práctica alimenticia en el pasado. El hecho de estar alejados del control familiar contribuye también a que los estudiantes foráneos de origen social elevado revaloricen incidentalmente una práctica de origen popular'.

\section{La internacionalización de una práctica alimenticia: el caso de los tacos "al pastor"}

A partir del caso de los tacos "al pastor" trataremos de poner de relieve la llamada "internacionalización" de la comida mexicana. Gracias a este tipo de tacos vemos la importancia del origen extranjero de ciertos productos o la importancia de su consagración internacional para su legitimación nacional. La acogida de los llamados tacos "árabes", cuyo origen en México se remonta a Irak y a Líbano principalmente, benefició de ciertas condiciones favorables a su recepción: la "mexicanización" de la comida extranjera gozó de las expectativas positivas generadas por una oferta extranjera similar a la oferta gastronómica local. El "taco árabe" se convirtió en el llamado taco "al pastor" gracias a ciertos rasgos comunes entre ambas ofertas alimenticias. Su éxito radica en parte en su semejanza con los tradicionales tacos mexicanos y en la capacidad de la cocina mexicana para adaptar las influencias extranjeras. Las afinidades electivas entre los consumidores mexicanos y esta gama de productos extranjeros análogos a los tacos, hicieron posible su adopción en México. Los tacos "al pastor", también llamados "de trompo" en el norte del país, son uno de los ejemplos más emblemáticos del sincretismo culinario que caracteriza a la cocina mexicana.

Sr. César Rodríguez: Los "regios” [oriundos de Monterrey] ¡casi no conocen el taco griego [gyro]! Y los estudiantes, sí. Que he andado en Europa, que en España, en Estados Unidos.... Lo "checan" [comprueban] y dicen: ¡es igual [al gyro]! ¡Hasta sabe más rico!

Sr. Reyna (Taquería La Playita): ¡A mi me lo platicaron! Un señor que viajaba a la $\mathrm{Cd}$. de México me platicó como estaba [era]. Y de pláticas, sin haberlos visto yo nunca ni nada [los tacos], hice el aparato. Porque él me platicó como. Nomás que hicimos un "aparatón" así, grandote. Luego lo tuve que reformar yo, porque era mucha la lumbre que echaba. Y ahí empezamos (1964). Pero, no nos dimos a conocer masivamente, $¡$ sino hasta un año o dos años

\footnotetext{
9 Aunado a esto, aparece el servicio de "venta de alimentos" a domicilio, lo que representa una solución ideal para satisfacer la necesidad de alimentación de una comunidad de fuerte poder adquisitivo y poco proclive a las artes culinarias.
} 
después de eso! [Fue gracias a]... una exposición de Monterrey [Exposición Agrícola y Ganadera de Nuevo León], de Nuevo León. Vinieron unos muchachos de.... Parece que eran de, Puebla... Y trajeron esos tacos con el nombre de i"Doneraki”! Yo les decía tacos "al pastor", desde antes. Y ellos llegaron con que se llamaban Doneraki. ¡DONERAKI!, así solo, en una sola palabra. Un nombre que es, no sé si es europeo, o por allá. Quien sabe de que parte sea [ese nombre]. ${ }^{10}$

Sr. Javier Lozano (Taquis Tacos): Nosotros les llamamos [tacos] "de trompo" por la forma [del espetón donde se asa la carne]. De hecho, se conocen como [tacos] "al pastor" en [la Ciudad de] México. ¿Sabes de dónde viene la idea del trompo [del espetón]? Más que nada, fue así. Los tacos estilo árabe llegan a México, a Puebla. De ahí nace. El estilo árabe lleva carnero, puerco, no se que otra carne... tres tipos de carne lleva. Entonces, en México lo adaptan con puerco. Pero la forma de hacerlo es árabe.

La comunidad libanesa efectivamente introdujo los llamados tacos “árabes” en México en la década de los 1920-30s (Pilcher, 1998, p. 136; Pilcher, 2006a; Díaz \& Macluf, 2002). Los tacos "al pastor" no son otra cosa que la mexicanización del shawarma cuyo origen se remonta al Medio Oriente (Suremain, 2008, p. 26). El hecho que sean productos de origen extranjero modifica su percepción siendo mejor valorados por la comunidad mexicana, lo que aumenta las posibilidades de recepción en México. El malinchismo, es decir el apego a lo extranjero, sería una de las condiciones que favoreció la adopción, no sin una cierta adaptación a la sazón local, de este tipo de bocados en México.

Los tacos al pastor ilustran nítidamente la influencia extranjera en la cocina mexicana. Los tacos más populares de México no son mexicanos como comúnmente se cree; o al menos no son completamente mexicanos. Basta detenerse un poco para analizar los ingredientes y la forma de prepararlos para comprender sus orígenes. La técnica usada para asar la carne de los tacos al pastor con un espetón es prácticamente idéntica a la turca o a la griega, para preparar los döner kebabs y los gryos, respectivamente. Las principales especies como el orégano y el comino son también de origen mediterráneo. La carne de cordero fue remplazada por la carne de cerdo de origen europeo. Entre los ingredientes mexicanos figuran únicamente el jugo de piña, los chiles guajillo y pasilla, el adobo a base de achiote y las tortillas de maíz (la mayoría son sazonadores). Sin embargo, estos últimos son suficientes para imprimirle

\footnotetext{
${ }^{10}$ La familia Tabe, originaria de Irak, fue la que desarrolló el concepto de los llamados "tacos árabes" en México bajo el nombre de Tacos "Don Eraki".
} 
un matiz nacional a la comida venida de otros países. Vemos que no se trata solamente de la adopción de un bocado extranjero tal cual. Los tacos al pastor son más bien emblemáticos de la fusión de diferentes tradiciones culinarias. No es un azar que esta variedad de tacos sea la que conoció el mayor éxito comercial. Su popularidad se explica quizás por su pragmatismo. La sencillez de su preparación hace relativamente fácil adaptarlos a la estandarización que caracteriza la comida rápida. De ahí que sean prácticamente los únicos que existan bajo el modelo de franquicias (Tacos Inn, Tizoncito, Fogoncito, etc.). Símbolos del sincretismo culinario latinoamericano, los tacos al pastor son los que cruzan más fácilmente las fronteras sociales convirtiéndose en los más vendidos en México (muy populares también en Estados Unidos).

La percepción que se tenía de los tacos en México se fue modificando gracias a la expansión del comercio de tacos en Estados Unidos. Observamos que la globalización del mercado de alimentos (Pilcher, 2008b; Fumey, 2007; Sanchez, 2007 ${ }^{11}$ ) contribuyó a eliminar algunos de los estigmas negativos asociados al consumo de tacos (comida de pobres, riesgosos, insalubres), que como sabemos eran claramente plebeyos, campesinos, en fin, populares. El caso de los tacos en California demuestra magistralmente la manera en que estos llegaron a Estados Unidos con las olas de emigrantes que trajeron consigo las costumbres y las prácticas alimenticias de su tierra natal. Este ejemplo sugiere que los tacos perdieron su estigma de "clase" (baja) cuando cruzaron la frontera (Pilcher, 2008a, p. 31). Los tacos se inscribieron en una dinámica de internacionalización que va más allá de las fronteras nacionales gracias a la transferencia culinaria efectuada por los mexicanos instalados en California. Esta dinámica esclarece paradójicamente el proceso de reapropiación nacional que conocieron a través de su propia transnacionalización ${ }^{12}$.

Los tacos comercializados en Estados Unidos eran asociados a la "comida étnica" y conocieron un éxito considerable difundiéndose en forma de franquicias (Gabaccia, 1998, p. 167). Los tacos entraron al mercado de la restauración rápida, permitiendo diversificar su clientela sin que ésta tuviera la necesidad de cruzar las barreras segregacionistas de los años 1950s (Pilcher, 2008a, p. 26) y sin la necesidad de cruzar la frontera mexicano-americana. El prestigio que los tacos alcanzaron en el mercado estadounidense parecer

${ }^{11}$ La pizza representa la comida transcultural por excelencia. El (re)descubrimiento de la pizza por parte de los soldados norteamericanos en Italia, durante la segunda guerra mundial, contribuyó a cambiar la imagen que se tenía de ella en Estados Unidos (comida "para pobres"). La pizza se convierte con el tiempo en plato principal y encarna de cierta forma la identidad italiana (Sanchez, 2007, p. 65-67).

12 Aunque durante el proceso hayan sufrido una evidente desnaturalización. Los tacos "suaves" fueron sustituidos por los "tacos duros" a base de tortilla frita en aceite. 
haber contribuido a modificar positivamente la imagen que se tenía de ellos, tanto en Estados Unidos como en México. Lo cual no significa por lo tanto que esto les garantizase un éxito sistemático en su país de origen. No obstante, su consagración internacional nos permite comprender mejor la legitimación que los tacos conocieron posteriormente en México.

El descubrimiento por parte de la comunidad mexicana de las cadenas de comida rápida norteamericanas que vendían tacos (Del Taco, Taco Palace, Taco Time, Taco Bell, etc.) contribuyó a transformar la representación que se tenía de ellos hasta entonces. El ir y venir de "braceros", jornaleros y otros emigrantes temporales también ayudó a modificar la representación de esta práctica alimenticia. El fallido intento de Taco Bell por conquistar el mercado mexicano en 1992 fue otro factor decisivo para cambiar la calidad social de un alimento nacional ${ }^{13}$ (aunque modificando su naturaleza intrínseca) ${ }^{14}$. Independientemente de haber sido un fracaso económico, la irrupción de Taco Bell en México cambió la percepción del taco abriendo nuevas perspectivas de mercado, especialmente para el nicho representado por la clase media. La difusión de una imagen menos estigmatizada generó expectativas positivas para un mercado en plena expansión. A pesar del fracaso norteamericano, muchos mexicanos decidieron apostarle a este prometedor segmento alimenticio como lo atesta la aparición de un gran número de taquerías en ese periodo. La crisis de 1994-95 fue otro de los factores que dificultó la expansión de Taco Bell en México. Sin embargo, la delicada coyuntura económica favoreció la proliferación de taquerías mexicanas (García, 2007). El efecto de la crisis, aunado al relajamiento de los patrones de consumo, estimuló la emergencia de taquerías (restaurantes y puestos callejeros) que representan una alternativa alimenticia bastante competitiva.

\section{Conclusión}

El proceso de internacionalización participa en la definición de la legitimidad de los tacos a través de una forma de patrimonialización de

${ }^{13}$ El taco presenta ciertas semejanzas con la pizza. Plato étnico por excelencia (es decir una especialidad culinaria valorizada por una comunidad en el sentido que representa e integra la cultura del grupo), la pizza hace parte de las estrategias de grupos desplazados para mantener sus prácticas alimenticias en un contexto adverso donde su identidad se ve fragilizada. La pizza sufre en el extranjero (Francia y Estados Unidos) un cambio de estatus para convertirse en un signo de identificación pública y de identidad (Sanchez, 2007, p. 47)

${ }^{14}$ La compañía de comida rápida Taco Bell, a su vez subsidiaria de Pepsico, fracasó al querer venderle tacos a los mexicanos en su tierra. Las razones de tal revés son múltiples. Sin embargo, es de notar que esta franquicia no vendía tacos, sino una variante "americanizada" de los originales tacos mexicanos. Contrariamente a la tradición original, el sabor de los tacos de Taco Bell es agridulce y se acompañan de crema, mayonesa, ketchup y papas fritas. 
este alimento. El hecho de pertenecer al panteón gastronómico nacional los convierte en emblemas nacionales. Su consumo masivo desde los años 1950 los vuelve una práctica alimenticia difundida prácticamente en todos los grupos sociales. El consumo de tacos se divulgó fuertemente después de su internacionalización en el circuito norteamericano. El origen extranjero ${ }^{15}$ del taco al pastor también contribuyó a banalizar su imagen genérica entre la clientela mexicana. La circulación internacional del taco modificó su calidad social sin por lo tanto cambiar su naturaleza física. Conforme fue perdiendo su estigma popular, el taco fue ganando prestigio entre los consumidores y se expandió a otros nichos de mercado.

La patrimonialización y la internacionalización se complementan en la revalorización de los tacos. La primera fortalece la imagen de ser un producto auténtico y la segunda contribuye a eliminar sus aspectos negativos. La nueva representación que adquirió gracias a este doble proceso aceleró la generalización de su consumo en todo el espectro social. La reclasificación social del taco no puede entenderse si hacemos abstracción de las mediaciones sociales (emigrantes, taqueros, jóvenes) que tuvieron un papel decisivo en su difusión y promoción. La aparición de nuevos estilos de vida y el relajamiento de los hábitos alimenticios contribuyeron a que los tacos franquearan las fronteras sociales convirtiéndose en un elemento de la identidad mexicana. Las prácticas alimenticias esclarecen la forma en que un platillo refuerza su prestigio nacional que adquiere al circular por el extranjero. El taco atraviesa las fronteras sociales gracias a un complejo proceso de reconocimiento y legitimación que obedece a factores internos y externos.

\section{Referencias}

ANDERSON, Benedict. Imagined Communities. Reflections on the origin and spread of nationalism. London: Verso Press, 1991.

BEAUD, Stéphane; WEBER, Florence. Guide de l'enquête de terrain. Produire et analyser des données ethnographiques. Paris: La Découverte, 2003.

BRUEGEL, Martin; LAURIOUX, Bruno (Org.). Histoire et identités alimentaires en Europe. Paris: Hachette, 2002.

DIAZ, Martha; MACLUF, Lourdes. De Líbano a México. La vida alrededor de la mesa. México: Impresos Castellanos, 2002.

${ }^{15}$ De la misma forma que los reyes o monarcas de origen extranjero, cuya legitimidad radica en la insuficiencia (de prestigio, de peso histórico o de antigüedad de linaje) de los autóctonos (Sahlins, 2010), los tacos ganan fuerza de imposición por efecto de las propiedades o las características positivas obtenidas en el exterior. 
FOURNIER, Dominique. Deux ou trois choses que nous offre le soleil... Le repas au Mexique. In: FLANDRIN, Jean-Louis; COBBI, Jane (Org.). Tables de hier et tables d'ailleurs. Histoire et ethnologie du repas. Paris: Odile Jacob, 1999. p. $455-78$.

FUMEY, Gilles. La mondialisation de l'alimentation. L'information géographique, France, n. 2, p. 71-82, 2007.

GABACCIA, Donna. We are what we eat. Ethnic food and the making of americans. Cambridge: Harvard University Press, 1998.

GARCIA, Domingo. Una etnografía económica de los tacos callejeros en México. El caso de Monterrey. Estudios Sociales, México (en prensa).

GARCIA, Domingo. L'entreprenariat informel. Le cas des marchands de tacos à Monterrey (Mexique), Paris: EHESS, thèse (dir. Remi Lenoir), 2009.

GARCIA, Domingo. Réduction des activités économiques informelles au Mexique par le biais de l'entreprenariat à petite échelle. Colloque: Économie informelle, travail au noir. Marne-la-Vallée, France, 17 sept. 2007.

GARCIA, Domingo. Nuevas prácticas emprendedoras de una ciudad industrial. El caso de los "tacos" y los "antros" en Monterrey. $1^{\circ}$ Congreso Latinoamericano de Antropología, Argentina, p. 11-15, julio 2005.

HEILBRON, Johan. Échanges culturels transnationaux et mondialisation. Quelques réflexions. Regards Sociologiques, France, n. 22, p. 141-154, 2001.

HEINICH, Nathalie. La fabrique du patrimoine. De la cathédrale à la petite cuillère. Paris: Editions de la Maison de sciences de l'homme, 2009.

HOBSBAWN, Eric; RANGER, Terence. The Invention of Tradition. Cambridge: Cambridge University Press, 1992.

HUBERT, Annie. Cuisine et politique. Le plat national existe-t-il? Revue des sciences sociales, France, n. 27, p. 8-11, 2000.

PILCHER, Jeffrey. Que vivan los tamales! Food and the making of mexican identity. Albuquerque: University of New Mexico Press, 1998.

PILCHER, Jeffrey. ¡Tacos, joven! Cosmopolitismo proletario y la cocina nacional mexicana. Dimensión antropológica, México, v. 13, n. 37, p. 87-125, 2006 a.

PILCHER, Jeffrey. The sausage rebellion. Public health, private enterprises, and meat in Mexico City, 1890-1917. Albuquerque: University of New Mexico Press, 2006b.

PILCHER, Jeffrey. Was the taco invented in Southern California? Gastronomica: The journal of food and culture, California, v. 8, n. 1, p. 26-38, 2008a.

RIVRON, Vassili. Le goût de ces choses bien à nous. La valorisation de la samba comme emblème national. Actes de la recherche en sciences sociales, France, v. 181182, p. 126-141, 2010.

SAHLINS, Marshall. Stranger-kings and stranger kin. The alterity of power and vice versa. Paris: Conferencia en el EHESS, Francia, el 14 de abril de 2010.

SANCHEZ, Sylvie. Pizza connexion. Une séduction transculturelle. Paris: CNRS, 2007. 
SUREMAIN de, Charles-Édouard. Shawarmas contre McDo. Mondialisation et standardisation alimentaire à l'épreuve des contestations identitaires (Bolivie). Anthropology of food, France, S4, mayo, 2008. Disponible en: <http://aof.revues.org/ index3693.html>.

THIESSE, Anne-Marie. La création des identités nationales. Europe $17^{e}-20^{e}$ siècle. Paris: Seuil, 1999.

WEBER, Florence. Manuel de l'ethnographe. Paris: PUF, 2009.

WEBER, Florence; DUFY, Caroline. L'ethnographie économique. Paris: La Découverte, 2008. 